\title{
Mechanistic studies of cytotoxic activity of the mesoionic compound MIH 2.4Bl in MCF-7 breast cancer cells
}

\author{
LUCIANA AMARAL DE MASCENA COSTA ${ }^{1,2}$, DIPTI DEBNATH ${ }^{3}$, ASHLYN C. HARMON ${ }^{2}$, \\ SILVANY DE SOUSA ARAÚJO ${ }^{1}$, HELIVALDO DIÓGENES DA SILVA SOUZA ${ }^{4}$, \\ PETRÔNIO FILGUEIRAS DE ATHAYDE FILHO ${ }^{4}$, AUREA WISCHRAL ${ }^{5}$, \\ MANOEL ADRIÃO GOMES FILHO ${ }^{1}$ and J. MICHAEL MATHIS ${ }^{2,3}$
}

\begin{abstract}
${ }^{1}$ Department of Morphology and Animal Physiology, Federal Rural University of Pernambuco, Recife, PE 52171-900, Brazil;
${ }^{2}$ Department of Comparative Biomedical Sciences, Louisiana State University, Baton Rouge, LA 70803;

${ }^{3}$ Graduate School of Biomedical Sciences, University of North Texas Health Science Center, Fort Worth, TX 76107, USA;

${ }^{4}$ Department of Chemistry, Federal University of Paraíba, Cidade Universitária, João Pessoa, PB 58051-900;

${ }^{5}$ Department of Veterinary Medicine, Federal Rural University of Pernambuco, Recife, PE 52171-900, Brazil
\end{abstract}

Received January 19, 2020; Accepted May 13, 2020

DOI: $10.3892 / 01.2020 .11763$

\begin{abstract}
In the present study, the cytotoxic effects of a 1,3-thiazolium-5-thiolate derivative of a mesoionic compound, $\mathrm{MIH} 2.4 \mathrm{Bl}$, were assessed in the MCF-7 breast cancer cell line. The cytotoxic effects of MIH 2.4Bl were determined using a crystal violet assay. Using a dose-response curve, the $\mathrm{IC}_{50}$ value of $\mathrm{MIH} 2.4 \mathrm{Bl}$ was determined to be $45.8 \pm 0.8 \mu \mathrm{M}$. Additionally, the effects of MIH $2.4 \mathrm{Bl}$ on mitochondrial respiration were characterized using oxygen consumption rate analysis. Treating MCF-7 cells with increasing concentrations of $\mathrm{MIH} 2.4 \mathrm{Bl}$ resulted in a significant reduction in all mitochondrial respiratory parameters compared with the control cells, indicative of an overall decrease in mitochondrial membrane potential. The induction of autophagy by $\mathrm{MIH} 2.4 \mathrm{Bl}$ was also examined by measuring changes in the expression of protein markers of autophagy. As shown by western blot analysis, treatment of MCF-7 cells with MIH 2.4Bl resulted in increased protein expression levels of Beclin-1 and ATG5, as well as an increase in the microtubule-associated protein 1A/1B light chain 3B (LC3B)-II to LC3B-I ratio compared with the control cells. Microarray analysis of changes in gene expression following $\mathrm{MIH} 2.4 \mathrm{Bl}$ treatment demonstrated 3,659 genes exhibited a fold-change $\geq 2$. Among these genes, 779 were up-regulated, and 2,880 were down-regulated in cells treated with MIH 2.4Bl compared with the control cells. Based on the identity of the transcripts and fold-change of
\end{abstract}

Correspondence to: Dr J. Michael Mathis, Graduate School of Biomedical Sciences, University of North Texas Health Science Center, 3500 Camp Bowie Boulevard, Fort Worth, TX 76107, USA E-mail:michael.mathis@unthsc.edu

Key words: autophagy, breast cancer, cancer therapy, chemotherapy, cytotoxicity, mesoionic compound, mitochondria expression, six genes were selected for verification by reverse transcription-quantitative (RT-q)PCR; activating transcription factor 3, acidic repeat-containing protein, heparin-binding EGF-like growth factor, regulator of G-protein signaling 2, Dickkopf WNT signaling pathway inhibitor 1 and adhesion molecule with Ig like domain 2 . The results of RT-qPCR analysis of RNA isolated from control and MIH 2.4Bl treated cells were consistent with the expression changes identified by microarray analysis. Together, these results suggest that $\mathrm{MIH} 2.4 \mathrm{Bl}$ may be a promising candidate for treating breast cancer and warrants further in vitro and in vivo investigation.

\section{Introduction}

Based on an analysis of data from the World Health Organization, breast cancer is the most common type of cancer and affects $\sim 2.1$ million women each year (1). In 2018, it is estimated that $\sim 634,000$ women worldwide died of breast cancer, accounting for $\sim 15 \%$ of all cancer-associated deaths among women (1). While early-stage breast cancer has been treated with relatively high success rates, advanced stage breast cancer remains a challenging disease to manage due to limitations of currently available therapies (2). To decrease the number of deaths, the development of new potent anticancer drugs is necessary. However, most chemotherapeutics used to treat cancer show cytotoxicity to both normal cells and cancer cells (3). Therefore, new drugs are required with reduced cytotoxicity towards normal cells and increased selectivity for cancer cells (3).

There has been a growing interest in recent years in the study of the anti-cancer activity of mesoionic compounds (4), which contain a five-carbon heterocyclic aromatic ring possessing a possessing sextet configuration of electrons (5). The heterocyclic ring is associated with a positive charge with a counterbalancing negative charge covalently located on an attached atom (5). This characteristic of a mesoionic structure having distinct separation of positive and negative charges configured on a heterocyclic ring structure, suggests the 
capability of mesoionic compounds to interact with biological polymers such as carbohydrates, lipids, nucleic acids, and proteins (6). While mesoionic compounds have internal positive and negative charges, their overall neutral charge allows them to cross the cell membranes.

The potential use of these compounds as cancer chemotherapeutic agents has been investigated primarily in the context of melanoma $(7,8)$. Treatment of melanoma cells with a 1,3,4-thiadiazolium mesoionic compound (MI-D) resulted in significant decreases in viability and proliferation. MI-D acts as an uncoupler of oxidative phosphorylation in the mitochondria by inhibiting the electron transport chain between complex II and complex III, resulting in a collapse of the transmembrane proton gradient and stimulating ATPase activity in the mitochondria (9). This disruption in mitochondrial energy appears to be associated with increased membrane permeability and fluidity (10).

Sydnones are mesoionic compounds containing a 1,2,3-oxadiazole core with a keto group in the 5 position. A series of sydnone derivatives were synthesized by Dunkley and Thoman (11). In screening a panel of cancer cell lines (MCF-7, NCI-H460, and SF-268 cells), an N-(4'-F-3'-nitrophenyl) sydnone was shown to exhibit significant cytotoxic activity (11). The anticancer activity of another sydnone compound, Sydnone 1 (SYD-1), was tested in vitro (12) and in vivo in using a rat Walker-256 carcinosarcoma model (13). Treatment of rats with SYD-1 decreased tumor volumes and tumor weight compared with the untreated animals (13). Investigation of the underlying mechanism of action of SYD-1 suggested that the anticancer activity of mesoionic compounds may be associated with changes in mitochondrial metabolism and activation of apoptotic pathways resulting in tumor cell death (12). The results of these studies confirm the potential role of mesoionic compounds as anticancer agents in the treatment of several types of cancer.

In our previous study, a 1,3-thiazolium-5-thiolate derivative of a mesoionic compound, MIH 2.4Bl was synthesized (14), and its potential selective anti-cancer properties were assessed using a panel of breast cancer cell lines and cells derived from normal human breast lineage. Treatment with MIH 2.4B1 inhibited growth of most of the breast cancer cell lines tested compared with normal human mammary epithelial cells. A focus was placed on the MCF-7 cell line as it is one of the most widely used cell lines as a model for hormone-receptor-positive breast cancer both in vitro and in vivo (15). The MCF-7 cell line is associated with differentiated features such as hormone receptor expression patterns typical of mammary epithelial cells (16), and its characterization also includes detailed transcriptome analysis $(17,18)$. Additionally, the extensive body of published literature on MCF-7 cells provides contextual relevance in studying breast cancer biology and drug development $(15,16)$. Treatment of MCF-7 cells with MIH 2.4Bl resulted in alterations in cell cycle distribution with an increased proportion of cells in the $\mathrm{G} 2 / \mathrm{M}$ phase compared with untreated cells. MCF-7 cells treated with MIH 2.4Bl also showed morphological changes consistent with apoptotic cell death, a finding confirmed by the results of a TUNEL assay.

The present study was designed to investigate further how the mesoionic compound MI 2.4Bl induced cell death in MCF-7 cells and explore possible underlying mechanisms of action. One such mechanism associated with mesoionic compounds is its effects on mitochondrial function (19), an organelle that has been functionally targeted in cancer treatment (20). Importantly, cells have a mechanism to protect against dysfunctional mitochondria, which can be deleterious to normal function. This mechanism, known as autophagy, involves the selective segregation and subsequent degradation of defective mitochondria before the onset of programmed cell death (21). In cancer, the autophagic process can be both pro- and anti-apoptotic, dependent on external stimuli and alterations in the microenvironment. Based on these considerations, the aim of the present study was to assess the cytotoxic effects of MIH 2.4 $\mathrm{Bl}$ on the mitochondria in MCF-7 cells, to understand the effects of the compound on autophagic cell death and to identify the genes involved in the process of cell death and proliferation following treatment.

\section{Materials and methods}

Synthesis of $\mathrm{MIH} 2.4 \mathrm{Bl}$. All reagents and solvents for the synthesis of MIH 2.4Bl were purchased from Sigma-Aldrich; Merck KGaA, and used without further purification. The method for synthesis of the free base mesoionic compound MIH 2.4Bl [chemical formula, 2-(4-chlorophenyl)-3-methyl 4-(4-methylphenyl)-1,3-thiazolium-5-thiolate], has been previously described (22). The chemical identity of the product was confirmed as previously described (22) by Fourier-transform infrared spectroscopy using an IFS 66 instrument (Bruker Corp.) and nuclear magnetic resonance spectroscopy using an Avance Ultrashield NMR spectrophotometer (Bruker Corporation) at $300 \mathrm{MHz}$ for ${ }^{1} \mathrm{H}$ and $75 \mathrm{MHz}$ for ${ }^{13} \mathrm{C}$.

Cell line and culture of cells. MCF-7 cells were obtained from American Type Culture Collection (ATCC) and maintained in DMEM (Genesee Scientific) supplemented with 10\% FBS (Gemini Bio-Products), $1 \%$ non-essential amino acids (Gemini Bio-Products), and $1 \%$ antibiotic/antimycotic solution (Gemini Bio-Products) containing $100 \mathrm{IU} / \mathrm{ml}$ penicillin, $100 \mu \mathrm{g} / \mathrm{ml}$ streptomycin and $25 \mu \mathrm{g} / \mathrm{ml}$ amphotericin B. Primary human mammary epithelial cells (HMECs) were obtained from ATCC, and cultured in Mammary Epithelial Cell Basal Medium (ATCC) supplemented with a Mammary Epithelial Cell Growth kit (ATCC) containing $5 \mu \mathrm{g} / \mathrm{ml} \mathrm{hH}$-insulin, $6 \mathrm{mM}$ L-glutamine, $0.5 \mu \mathrm{M}$ epinephrine, $5 \mu \mathrm{g} / \mathrm{ml}$ apo-transferrin, $5 \mathrm{ng} / \mathrm{ml} \mathrm{rH}-\mathrm{TGF}-\alpha, 0.4 \%$ ExtractP and $100 \mathrm{ng} / \mathrm{ml}$ hydrocortisone hemisuccinate. MCF-10A cells were obtained from ATCC and cultured in DMEM/Ham's F12 media supplemented with 5\% Equine Serum (Gemini Bio-Products), $20 \mathrm{ng} / \mathrm{ml}$ epidermal growth factor (Sigma-Aldrich; Merck KGaA), $10 \mu \mathrm{g} / \mathrm{ml}$ insulin (Sigma-Aldrich; Merck KGaA), $0.5 \mathrm{mg} / \mathrm{ml}$ hydrocortisone (Sigma-Aldrich; Merck KGaA), $100 \mathrm{ng} / \mathrm{ml}$ cholera toxin (Sigma-Aldrich; Merck KGaA) and 1\% antibiotic/antimycotic solution. All cells were maintained in $5 \% \mathrm{CO}_{2}$ at $37^{\circ} \mathrm{C}$ in a humidified incubator.

Cytotoxicity assay. For the evaluation of cytotoxicity, a crystal violet assay was used as described previously (23). The crystal violet assay uses a non-specific dye whose staining pattern is directly proportional to the number of viable adherent cells. A total of $5 \times 10^{3}$ cells were seeded and cultured for $24 \mathrm{~h}$ in 
96-well tissue culture plates. Subsequently, the cells were treated with eight different concentrations of $\mathrm{MIH} 2.4 \mathrm{Bl}$ : $150 \mu \mathrm{M}$ and seven serial dilutions (resulting in 75, 37.5, 18.8, 9.4, 4.7, 2.3, and 1.2 $\mu \mathrm{M}$, respectively). As a positive control, cells were treated with $1.34 \mu \mathrm{M}$ doxorubicin, a concentration representing $\sim \mathrm{x} 10$ the reported $\mathrm{IC}_{50}$ in MCF-7 cells (24-26). Cells were treated with vehicle (DMSO) alone at $0.1 \%(\mathrm{v} / \mathrm{v})$ in media as a negative control. The cells were treated at each concentration using eight replicate wells for $72 \mathrm{~h}$. At each time point, the media was removed, and the wells were washed twice with Dulbecco's PBS (Gemini Bio-Products). Subsequently, the plates were gently inverted on filter paper to remove any remaining liquid and dried at room temperature. The dried cells were fixed with $20 \%$ methanol for $30 \mathrm{~min}$ at room temperature and stained with $0.5 \% \mathrm{w} / \mathrm{v}$ crystal violet solution for $5 \mathrm{~min}$ at room temperature. The plates were washed of excess crystal violet with deionized water, and the washed plates were air-dried. Subsequently, the crystal violet stained cells were solubilized with $200 \mu \mathrm{l}$ of $95 \%$ ethanol/40 mM HCl for $30 \mathrm{~min}$ at room temperature. The optical density (OD) of each well was measured at $595 \mathrm{~nm}$ using a SpectraMax 190 absorbance microplate reader (Molecular Devices, LLC).

Oxygen consumption rate (OCR) measurements. OCR measurements were performed using a Seahorse XF96 Extracellular Flux Analyzer (Seahorse Bioscience). MCF-7 cells were initially seeded at a density of $5 \times 10^{3}$ cells per well into Seahorse 96-well microplates for $24 \mathrm{~h}$. Subsequently, the cells were treated for $24 \mathrm{~h}$ with MIH 2.4Bl using the eight aforementioned concentrations, and the same positive and negative controls were used as described above. After treatment, the cell media was changed to unbuffered DMEM containing $20 \mathrm{mM}$ carnosine and $2.0 \mathrm{mM}$ sodium pyruvate. The MCF-7 cells were maintained at $37^{\circ} \mathrm{C}$ under normal atmospheric conditions. After baseline measurements, OCR was measured following the addition of $1 \mu \mathrm{M}$ oligomycin, then $1 \mu \mathrm{M}$ FCCP, and finally $1 \mu \mathrm{M}$ rotenone $/ 5 \mu \mathrm{M}$ antimycin A to each well. After baseline measurements, the ATP-linked OCR was determined as the measurements after inhibition of ATP synthase by oligomycin. FCCP (an uncoupler of mitochondrial oxidative phosphorylation) was added to determine maximal respiration. The maximal respiration was normalized by subtracting the measurement of the non-mitochondrial respiration, which was identified by addition of rotenone/antimycin A. The basal respiration was subtracted from the maximal respiration to obtain the reserve respiratory capacity. OCR data was reported in units of picomoles per minute and was generated using the Wave Desktop and Controller Software (version 2.6.1; Seahorse Bioscience).

Western blot analysis. MCF-7 cells were seeded in $60 \mathrm{~mm}$ tissue culture dishes at a density of $3 \times 10^{6}$ cells/dish for $24 \mathrm{~h}$. Subsequently, the cells were treated for 48 or $72 \mathrm{~h}$ with $75 \mu \mathrm{M}$ MIH 2.4Bl. The MCF-7 cells were also treated with vehicle (DMSO) alone at $0.1 \%(\mathrm{v} / \mathrm{v})$ in media as a negative control. After each treatment time point, the cells were harvested by scraping into ice-cold RIPA buffer (composed of $50 \mathrm{mM}$ Tris, $\mathrm{pH} 8.0,150 \mathrm{mM} \mathrm{NaCl}, 1 \% \mathrm{NP}-40,0.5 \%$ deoxycholate, and $0.1 \%$ sodium dodecyl sulfate, containing a cocktail of protease inhibitors (Pierce Protease Inhibitor Tablets; Thermo
Fisher Scientific, Inc.). Total protein concentrations of the cell lysates were determined using a Bradford protein assay (Bio-Rad Laboratories, Inc.). Normalized protein samples (20 $\mu \mathrm{g}$ each) were denatured at $95^{\circ} \mathrm{C}$ using Laemmli sample buffer (Bio-Rad Laboratories, Inc.), separated by electrophoresis on $4-20 \%$ gradient polyacrylamide mini gels (Bio-Rad Laboratories, Inc.) and transferred using a Trans-Blot Turbo transfer apparatus (Bio-Rad Laboratories, Inc.) to PVDF membranes. The membranes were subsequently incubated at room temperature for $1 \mathrm{~h}$ in blocking buffer of TBS (composed of $50 \mathrm{mM}$ Tris-Cl, pH 7.5 and $150 \mathrm{mM} \mathrm{NaCl}$ ), containing $0.01 \%$ Tween-20 (TBS-T) and 5\% non-fat milk (w/v). Subsequently, the membranes were washed three times using TBS-T for 5 min each. The membranes were incubated overnight with gentle agitation at $4^{\circ} \mathrm{C}$ in $5 \mathrm{ml}$ blocking buffer containing mouse monoclonal anti-Beclin-1 (cat. no. 2A4 MA5-15825; 1:500; Invitrogen; Thermo Fisher Scientific, Inc.), rabbit polyclonal anti-ATG5 (cat. no. PA5-35201; 1:1,000; Invitrogen; Thermo Fisher Scientific, Inc.), rabbit polyclonal anti-LC3 II/I (cat. no. PA5-46286; 1:1,000; Invitrogen; Thermo Fisher Scientific, Inc.) or mouse monoclonal anti-GAPDH antibody (cat. no. GA1R, MA5-15738; 1:1,000; Invitrogen; Thermo Fisher Scientific, Inc.). The membranes were washed five times with TBS-T for 5 min each, and subsequently incubated with a goat polyclonal horseradish peroxidase-conjugated secondary anti-rabbit antibody (IgG; cat. no. A28177, 1:10,000; Invitrogen; Thermo Fisher Scientific, Inc.) or goat polyclonal anti-mouse antibody (IgG; cat. no. A28177; 1:10,000; Invitrogen; Thermo Fisher Scientific, Inc.) for $30 \mathrm{~min}$ at room temperature. Bands associated with specific proteins were detected using the SuperSignal West PICO plus chemiluminescent substrate (Thermo Fisher Scientific, Inc.) and analyzed using a ChemiDoc imaging system (Bio-Rad Laboratories, Inc.). Densitometry analysis was performed using Quantity One software (version 4.6.6; Bio-Rad Laboratories, Inc.).

Total RNA isolation. MCF-7 cells were cultured in 6-well tissue culture plates at a density of $1 \times 10^{6}$ cells per well and incubated for $24 \mathrm{~h}$. Subsequently, the cells were treated for $24 \mathrm{~h}$ with $75 \mu \mathrm{M}$ MIH 2.4Bl. As a control, cells were treated with vehicle (DMSO) alone at $0.1 \%(\mathrm{v} / \mathrm{v})$ in media. A Zymo Research Direct-Zol RNA Purification kit (Zymo Research Corp.) was used to extract and purify total RNA from each sample well for subsequent analysis. The quantity and integrity of the RNA samples were assessed using an Agilent Tape Station RNA Assay (Agilent Technologies, Inc.).

Microarray analysis. Total RNA samples were reverse transcribed using an Affymetrix 3'IVT Express kit (Affymetrix; Thermo Fisher Scientific, Inc.) with T7-Oligo(dT) primer, which contains a T7 RNA polymerase promoter. Then, second-strand cDNA synthesis was performed and was followed by antisense RNA (aRNA) amplification of the cDNA templates prepared using biotinylated nucleotide analogs according to the manufacturer's protocol. Biotin-labeled aRNA was fragmented by incubating the samples for $35 \mathrm{~min}$ at $94^{\circ} \mathrm{C}$ in reaction buffer (40 mM Tris-acetate, $\mathrm{pH} 8.1,100 \mathrm{mM}$ potassium acetate and $30 \mathrm{mM}$ magnesium acetate). Subsequently, $15 \mu \mathrm{g}$ of the fragmented aRNA was hybridized at $45^{\circ} \mathrm{C}$ for 
Table I. Target sequence identity of selected genes used in reverse transcription-quantitative PCR analysis.

\begin{tabular}{llcc} 
Gene name & Gene symbol & $\begin{array}{c}\text { Amplicon } \\
\text { length }\end{array}$ & Unique assay ID \\
\hline Activating transcription factor 3 & ATF3 & 76 & qHsaCEP0053273 \\
Acidic repeat-containing protein & ACRC & 149 & qHsaCIP0029745 \\
Adhesion molecule with Ig like domain 2 & AMIGO2 & 96 & qHsaCEP0052159 \\
Dickkopf WNT signaling pathway inhibitor 1 & DKK1 & 96 & qHsaCEP0050470 \\
Gyceraldehyde-3-phosphate dehydrogenase & GAPDH & 117 & qHsaCEP0041396 \\
Heparin-binding EGF-like growth factor & HBEGF & 117 & qHsaCEP0050730 \\
Regulator of G-protein signaling 2, 24 kDa & RGS2 & 112 & qHsaCEP0024158 \\
\hline
\end{tabular}

16 h to a Human Genome U133 Plus 2.0 Array (Affymetrix; Thermo Fisher Scientific, Inc.) in a GeneChip Hybridization Oven 645 (Thermo Fisher Scientific, Inc.). After the hybridization was complete, the gene chips were automatically washed and stained for expression detection with streptavidin-phycoerythrin in a GeneChip Fluidics Station 450 (Thermo Fisher Scientific, Inc.) using the FS450_0004 protocol. Finally, the probe arrays were scanned and processed using a GeneChip Scanner 3000 7G (Thermo Fisher Scientific, Inc.). Transcriptome Analysis Console version 4.0.2 (Thermo Fisher Scientific, Inc.) was used to analyze the fold-change of each gene based on the relative differences between signal intensities. Only genes with a fold-change $\geq 2.0$ were considered differentially expressed genes (DEGs).

The Database for Annotation, Visualization and Integrated Discovery (DAVID; david.abcc.ncifcrf.gov/) and analytics tools $(27,28)$ was used to map the DEGs to relevant pathway networks in the Gene Ontology (GO; geneontology.org/) and Kyoto Encyclopedia of Genes and Genomes (KEGG; genome. jp/kegg/pathway.html/) databases (29-31). The P-values were calculated by DAVID using the Benjamini-Hochberg method (32) to control the False Discovery Rate, $\mathrm{P}<0.05$ was considered to indicate a statistically significant difference. The top ontologies in the GO subsets (molecular functions, biological processes, and cellular components), as well as the top KEGG pathways, were selected based on a hierarchy of the P-values calculated.

Reverse transcription-quantitative $(R T-q) P C R$. Expression levels of select genes identified as up or downregulated following MIH 2.4Bl treatment compared with vehicle (control) cells based on the microarray analysis were quantified by RT-qPCR. A panel of four upregulated genes [activating transcription factor 3 (ATF3), acidic repeat-containing protein (ACRC), heparin-binding EGF-like growth factor (HBEGF), regulator of G-protein signaling 2 (RGS2)] and two downregulated genes [Dickkopf WNT signaling pathway inhibitor 1 (DKK1) and adhesion molecule with Ig like domain 2 (AMIGO2)] was used and compared to the expression of GAPDH as a control. A Verso 1-step RT-qPCR kit (Thermo Fisher Scientific) was used to synthesize the first-strand cDNA and perform quantitative PCR in a single step assay according to the manufacturer's protocol. An Applied Biosystems 7300 Real-Time PCR system (Thermo Fisher Scientific, Inc.) was used to detect the transcripts using PrimePCR primers and FAM-labeled probe mixtures (Bio-Rad
Laboratories, Inc.). The sequences of the primers and probes are not disclosed by the manufacturer; however, target sequence information is presented in Table I. The thermocycling conditions for the qPCR reactions were: $15 \mathrm{~min}$ at $50^{\circ} \mathrm{C}$ for the cDNA synthesis; initial denaturation at $95^{\circ} \mathrm{C}$ for $5 \mathrm{~min}$; followed by 40 cycles of $95^{\circ} \mathrm{C}$ for $15 \mathrm{sec}$ and $60^{\circ} \mathrm{C}$ for $60 \mathrm{sec}$. The comparative quantification cycle $(\mathrm{Cq})$ method was used to quantify the relative changes in gene expression (33).

Statistical analysis. The cell viability data were calculated from the mean OD readings obtained for each concentration of the MIH 2.4Bl mesoionic compound assayed normalized to the mean OD readings of the negative control and mean blank values, and is expressed as the mean percentage \pm the standard error of the mean. The dose-response curves were plotted and analyzed to calculate the $\mathrm{IC}_{50}$ values using a four parameters logistic (4PL) model for curve fitting, in which a variable slope was employed that allowed non-linear fitting of the equation. The $\mathrm{IC}_{50}$ values (concentrations that reduced cell viability to $50 \%$ compared with the negative control) were determined using nonlinear regression analysis in the GraphPad Prism version 7.0 (GraphPad Software, Inc.) and standard error of the mean was calculated from the cell viability data. The mean percent inhibition values were plotted against the $\mathrm{MIH} 2.4 \mathrm{Bl}$ concentrations allowing for the generation of a sigmoidal dose-response curve using the following equation: $\mathrm{Y}=100 /\left[1+10^{\wedge}\left(\left(\log \mathrm{IC}_{50}-\mathrm{X}\right) \times\right.\right.$ HillSlope $\left.\left.)\right)\right]$. The $\mathrm{IC}_{50}$ values were obtained by the interpolation of curve-fit data from each curve using the GraphPad Prism.

Replicates of eight OCR measurements are expressed as the mean \pm the standard error of the mean. Data from RT-qPCR analysis were calculated from three replicates. For the comparison between data, a Student's t-test was used. $\mathrm{P}<0.05$ was considered to indicate a statistically significant difference.

\section{Results}

Effect of MIH 2.4Bl on cellular cytotoxicity. To determine the cytotoxic effects of MIH 2.4Bl on the MCF-7 cells, a crystal violet assay was used, based on the simplicity of the assay and its suitability for examining the effects of chemotherapeutics on cell survival and growth inhibition (23). The dose-response curve presented in Fig. 1 indicates that MIH 2.4Bl decreased MCF-7 cell viability after $72 \mathrm{~h}$ treatment. Based on the curve fitting, the $\mathrm{IC}_{50}$ for $\mathrm{MIH} 2.4 \mathrm{Bl}$ was $45.9 \pm 1.0 \mu \mathrm{M}$. MCF-10A (a 
A

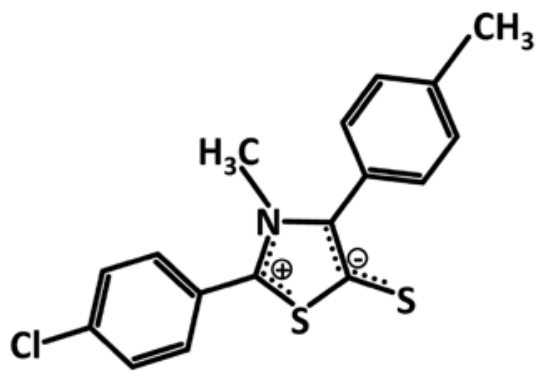

B

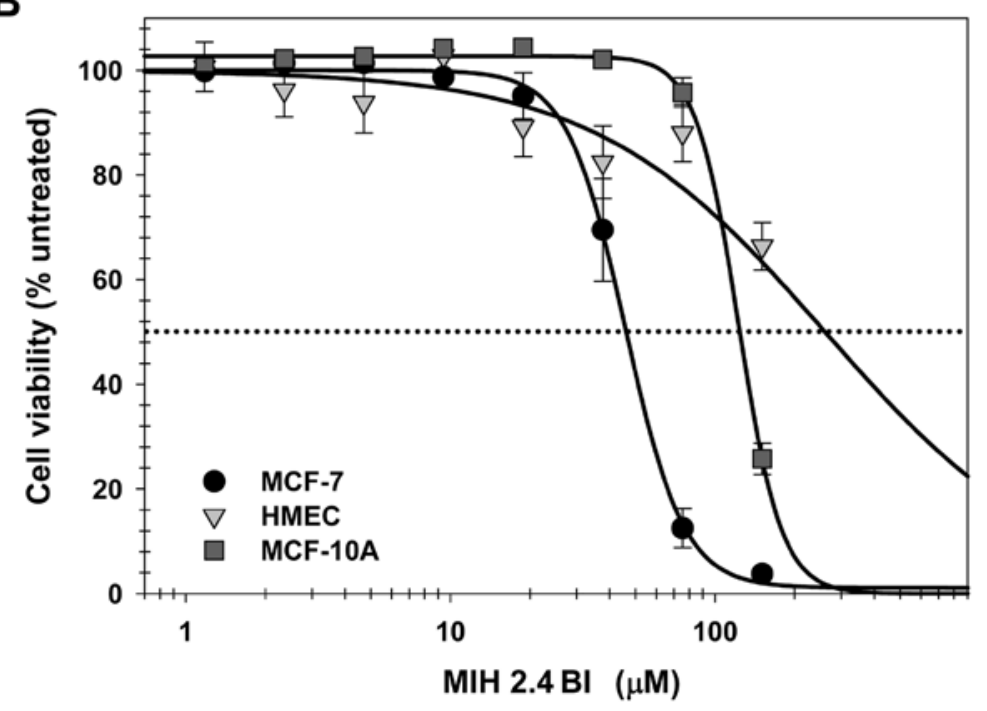

Figure 1. Effect of MIH 2.4Bl on the viability of MCF-7 breast cancer cells. (A) The chemical structure of the mesoionic compound MIH 2.4Bl. (B) Relative cell viability of MCF-7 cells (circles) was determined using a crystal violet assay after $72 \mathrm{~h}$ of treatment with increasing concentrations of MIH 2.4Bl. The relative cell viability of HMEC (triangles) and MCF-10A cells (squares) was also determined. Each data point represents the mean \pm the standard error of the mean of eight replicates. The $\mathrm{IC}_{50}$ values were calculated based on data from the best fit data of the Hill slope curve using a nonlinear regression analysis. HMEC, human mammary epithelial cells.

A

Beclin-1

ATG5

LC3B

GAPDH

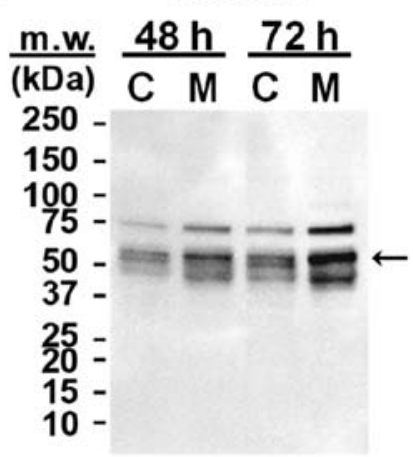

$48 \mathrm{~h} \frac{72 \mathrm{~h}}{\mathrm{C}}$

$48 \mathrm{~h} \underline{72 \mathrm{~h}}$

$48 \mathrm{~h} \underline{72 \mathrm{~h}}$ C M C M C M C M
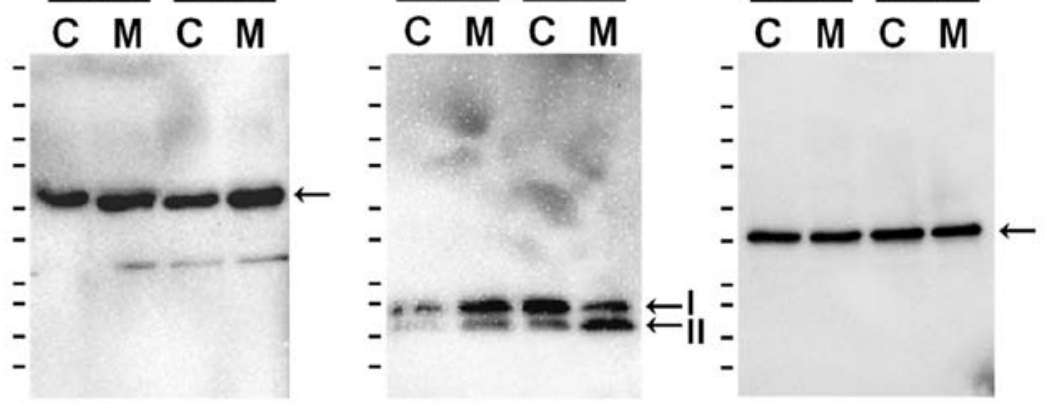

B
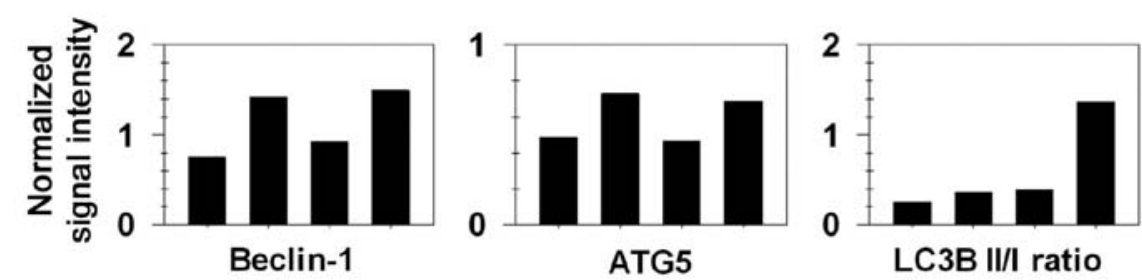

Figure 2. Effect of MIH 2.4Bl on the induction of autophagy in MCF-7 cells. (A) Expression of Beclin-1, ATG5, LC3B II/I and GAPDH were determined by western blot analysis in control or MIH 2.4Bl treated cells after 48 or $72 \mathrm{~h}$ of culture. (B) Densitometry analysis of Beclin-1, ATG5, LC3B II and LC3B I expression was performed and normalized to GAPDH. Data are presented as histograms of normalized Beclin-1 and ATG5 intensity values, and the intensity ratios of LC3B-II to LC3B-I. LC3B, microtubule-associated protein 1A/1B light chain 3B. C, control cells; M, MIH 2.4Bl treated cells. 

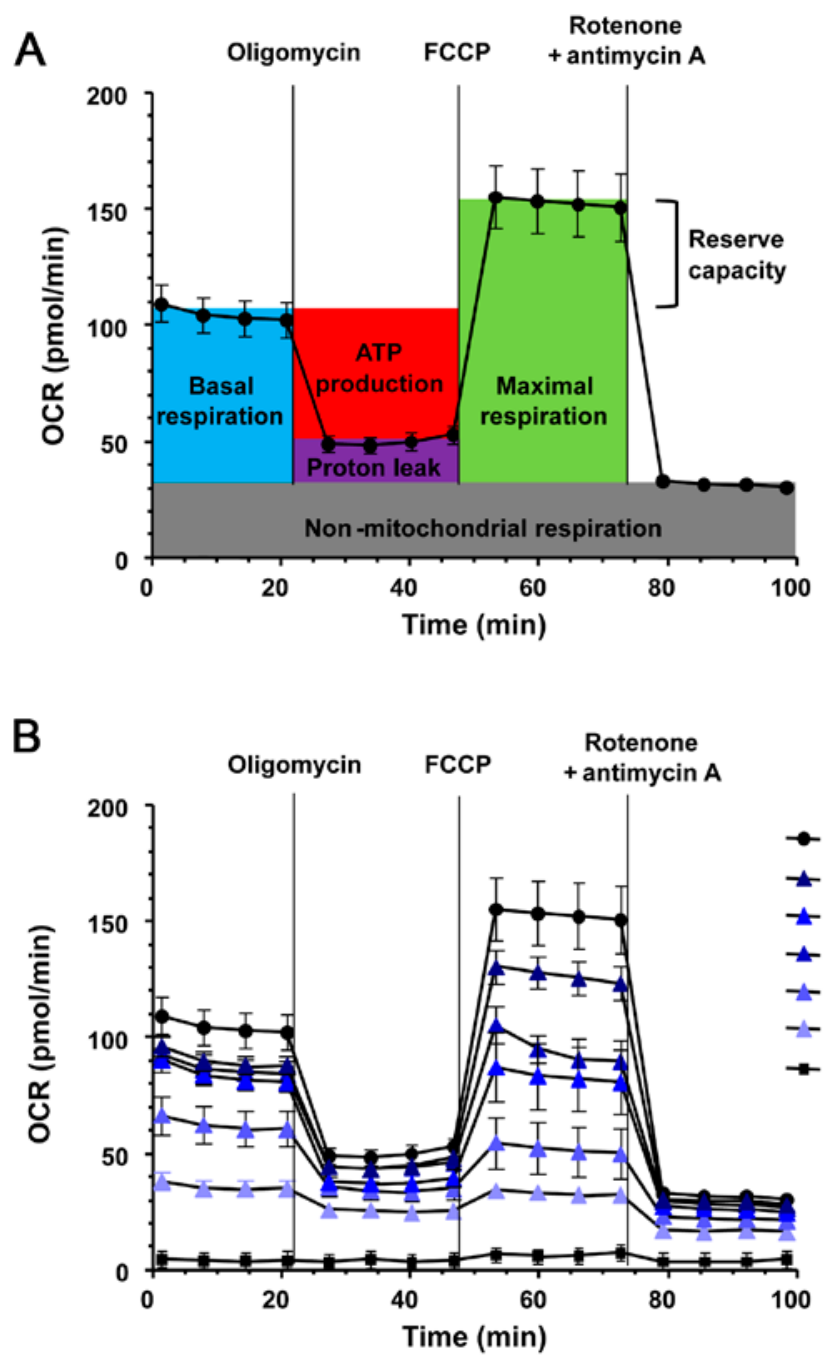

Figure 3. Dose-dependent effects of MIH 2.4Bl on the OCR of MCF-7 cells. (A) Representative schematic of OCR using specific inhibitors to assess mitochondrial function. After four initial measurements to acquire the baseline OCR, sequential injections of oligomycin, FCCP, and a rotenone/antimycin A mixture were performed with four OCR measurements obtained after each injection. Shown in the OCR profile are calculations of basal respiration, maximal respiration, ATP production, proton leak, reserve respiratory capacity and non-mitochondrial respiration. (B) OCR profiles were determined in MCF-7 cells after treatment for $24 \mathrm{~h}$ with 4.7, 9.4, 18.8,37.7 or $75.4 \mu \mathrm{M} \mathrm{MIH} 2.4 \mathrm{Bl}$. Treatment with $1.5 \mu \mathrm{M}$ doxorubicin for $24 \mathrm{~h}$ was used as a positive control for maximum cytotoxic effect. Each data point represents the mean \pm the standard error of the mean of eight replicates. OCR, oxygen consumption rate.

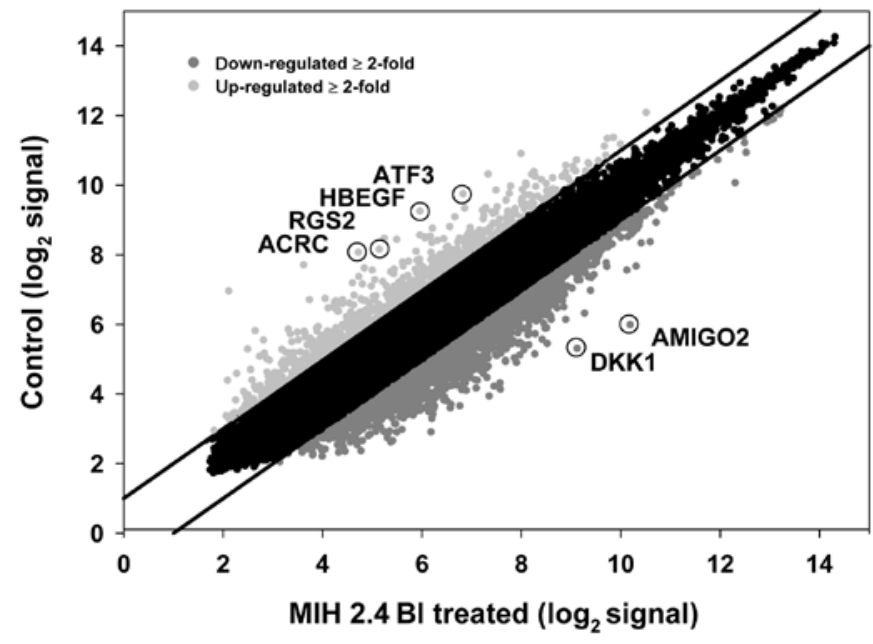

Figure 4. Microarray expression summary. Data are expressed as a scatter plot showing the $\geq 2$-fold upregulated and downregulated genes (light grey and dark grey dots, respectively) in MCF-7 cells treated with MIH 2.4B compared with control cells. Genes with $<2$-fold change are shown as black dots. spontaneously immortalized non-tumorigenic cell line derived from benign proliferative breast tissue) required a significantly higher concentration of $\mathrm{MIH} 2.4 \mathrm{Bl}$ required for inhibition of cell viability, with an $\mathrm{IC}_{50}$ of $123.8 \pm 1.0 \mu \mathrm{M}$. Primary HMEC only exhibited slight inhibitory activity following $\mathrm{MIH} 2.4 \mathrm{Bl}$ treatment, with an $\mathrm{IC}_{50}$ of $917 \pm 19 \mu \mathrm{M}$ (Fig. 1). These results demonstrate the potential cancer specificity of cytotoxic inhibition on MCF-7 cells mediated by MIH 2.4B1.

Effect of MIH 2.4Bl on the induction of autophagy. To determine the significance of autophagy in the treatment of MCF-7 cells with MIH 2.4B1, the expression of Beclin-1 and ATG5 were assessed due to their role in autophagy. As shown in Fig. 2A, western blotting analysis showed that Beclin-1 and ATG5 expression were increased following treatment with $\mathrm{MIH}$ 2.4B1. Microtubule-associated protein 1A/1B light chain 3B (LC3B) is a specific autophagy marker widely utilized for measuring the autophagy level in cells and tissues. As shown in Fig. 2A, both LC3B-I and LC3B-II were present as 18 and $16 \mathrm{kDa}$ bands, respectively. LC3B-I 
Table II. Identity of selected genes differentially regulated by MIH 2.4Bl treatment.

\begin{tabular}{lrlll}
\hline Probe Set ID & Fold-change & Gene symbol & \multicolumn{1}{c}{ Description } & Transcript ID \\
\hline 238825_at & 10.2 & ACRC & Acidic repeat containing & Hs.135167.0 \\
202672_s_at & 7.5 & ATF3 & Activating transcription factor 3 & g4502262 \\
203821_at & 9.8 & HBEGF & Heparin-binding EGF-like growth factor & g4503412 \\
202388_at & 8.1 & RGS2 & Regulator of G-protein signaling 2 & g4506516 \\
222108_at & -18.3 & AMIGO2 & Adhesion molecule with Ig-like domain 2 & Hs.121520.0 \\
204602_at & -14.0 & DKK1 & Dickkopf WNT signaling pathway inhibitor 1 & g7110718 \\
\hline
\end{tabular}

is the cytosolic precursor form of LC3B-II, whereas LC3B-II is an active form conjugated with phosphatidylethanolamine and is membrane-bound. A widely accepted indicator of the number of autophagosomes is an increase in the LC3B-II to LC3B-I ratio (34). As shown in Fig. 2B, there was a significant increase in the LC3B-II to LC3B-I ratio in MCF-7 cells treated for $72 \mathrm{~h}$ with MIH 2.4Bl. Although the LC3B levels increased in control cells between 48 and $72 \mathrm{~h}$, there was no increase in the ratio of LC3B-II to LC3B-I. These results suggest that induction of autophagy is a potential mechanism of cell death mediated by MIH 2.4B1.

Effect of $M I H 2.4 B l$ on mitochondrial OCR. Tumor cells are highly dependent on glycolytic metabolism since this bioenergetic pathway produces energy and metabolites capable of supporting the tumor's high growth and proliferation rates relatively quickly (35). Thus, measuring changes in mitochondrial OCR is a reliable approach for evaluating cellular energy metabolism (36). Therefore, the mitochondrial OCR was measured in MCF-7 cells following treatment with increasing concentrations of MIH 2.4 from 4.7-75.0 $\mu \mathrm{M}$ for $24 \mathrm{~h}$. Untreated MCF-7 cells were used as the negative control, and doxorubicin treated MCF-7 cells (at a concentration of $1.5 \mu \mathrm{M}$ ) were used as the positive control. The initial mitochondrial OCR was measured using a Seahorse XF96 analyzer and after sequential addition of oligomycin, FCCP, and a rotenone/antimycin A combination (Fig. 3A). These additions allowed for the determination of the following mitochondrial parameters: Basal respiration, maximal respiration, ATP production, proton leak, reserve respiratory capacity, and non-mitochondrial respiration. As shown in Fig. 3B, treatment of MCF-7 cells with increasing concentrations of $\mathrm{MIH} 2.4 \mathrm{Bl}$ resulted in a significant reduction in all the mitochondrial respiratory parameters compared with the control cells, indicative of an overall decrease in mitochondrial membrane potential. At 75.0 $\mu \mathrm{M}$ MIH 2.4Bl treatment, almost all mitochondrial respiration was abolished, leaving only residual oxygen consumption (non-mitochondrial respiration). These results highlight the potential of therapeutic targeting of mitochondrial metabolism using $\mathrm{MIH} \mathrm{2.4Bl}$ for cancer therapy.

Analysis of DEGs in MCF-7 cells treated with $\mathrm{MIH} 2.4 \mathrm{Bl}$. A DNA microarray analysis of mRNA levels in MCF-7 cells was performed following treatment with $\mathrm{MIH} 2.4 \mathrm{Bl}$ to determine changes in global genome-wide gene expression patterns. Microarray analysis of mRNA isolated from control and MIH 2.4Bl treated cells was performed using human Affymetrix Human Genome U133 Plus 2.0 Arrays. A scatter plot of the mean of the normalized gene expression of RNA from MIH 2.4Bl treated cells vs. RNA from control cells shows the DEGs (Fig. 4). A total of 3,659 DEGs were detected between the two groups with a fold-change $\geq 2.0$. Among these genes, 779 were upregulated and 2,880 were downregulated in cells treated with $\mathrm{MIH} 2.4 \mathrm{Bl}$ compared with the control cells (Tables SI and SII). There were 144 genes detected with a fold-change $\geq 5.0$, including 37 upregulated genes and 107 downregulated genes. Based on the identity of the transcripts and fold-change of expression, 6 genes were selected for verification by RT-qPCR, as shown in Table II.

$R T-q P C R$ analysis. The differences in the expression of these 6 genes between treated and untreated cells is shown in Fig. 5. RT-qPCR analysis showed consistent results with the results of the microarray analysis. ACRC, ATF3, HBEGF and RGS2 genes were significantly upregulated in cells treated with $\mathrm{MIH} 2.4 \mathrm{Bl}$ cells compared with the control cells. In contrast, expression levels of AMIGO2 and DKK1 genes were significantly downregulated in $\mathrm{MIH} 2.4 \mathrm{Bl}$ treated cells compared with the control cells. The RT-qPCR results confirmed the trend in changes of gene expression identified using microarray analysis, although the fold-differences detected were different between the two analyses.

To gain further insight into the pattern of gene expression changes following MIH 2.4Bl treatment, GO ontology analysis and KEGG pathway analysis were performed using DAVID. Of the 3,659 DEGs identified from the microarray analysis, 2,643 genes were analyzed further using DAVID; 290 were unable to be mapped with HUGO Gene Nomenclature Committee gene symbols, and 726 were duplicate genes that were ignored in favor of lower adjusted P-values. As shown in Fig. 6, the biological processes of the DEGs were primarily enriched DNA-template transcription as well as positive and negative regulation of transcription. The cellular components of the DEGs were primarily enriched in cytoplasm, nucleoplasm, and nucleus components. The molecular functions of the DEGs were identified to be primarily enriched in protein binding. The results of KEGG analysis showed 12 pathways were significantly enriched by the DEGs (Table III). Of these pathways, three involved mechanisms regulating cell signaling (hsa04068, hsa04550, and hsa04350), and two involved pathways regulating cancer (hsa05202 and hsa05211). The most significant pathway mediating FoxO signaling (hsa04068) serves a role 


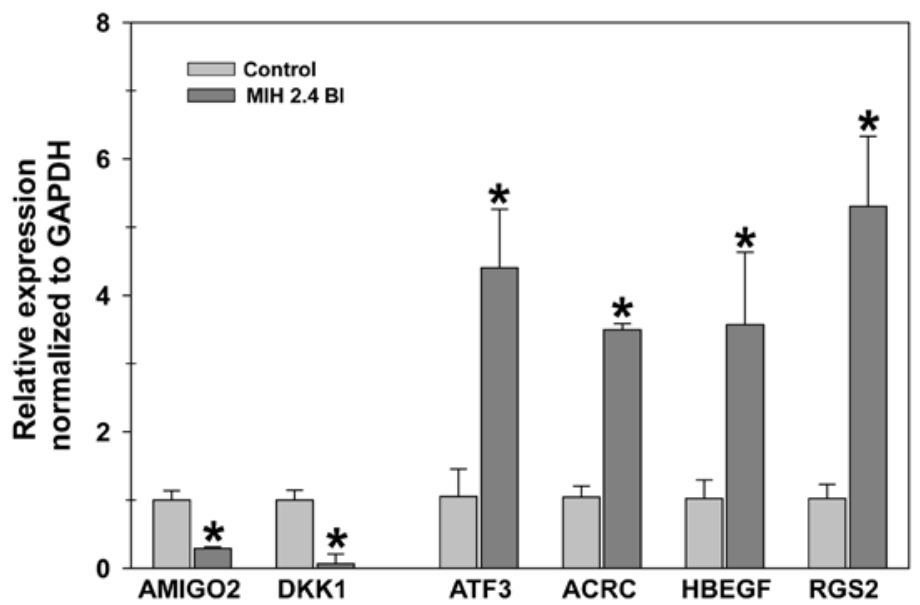

Figure 5. Effect of MIH 2.4Bl on the mRNA expression levels of six differentially expressed genes in MCF-7 cells. Relative expression levels of ACRC, HBEGF, ATF3, RGS2, DKK1 and AMIGO2 were determined in control and MIH 2.4B1 treated cells. Data were calculated as ${ }^{\Delta \Delta C q}$ values based on Cq expression levels in MIH 2.4Bl treated cells compared with control cells, and normalized to GAPDH expression. Each data point represents the mean \pm the standard error of the mean of three replicates. "P<0.05. ACRC, acidic repeat-containing protein; HBEGF, heparin-binding EGF-like growth factor; ATF3, activating transcription factor 3; RGS2, Regulator of G-protein signaling 2; DKK1, Dickkopf WNT signaling pathway inhibitor 1; AMIGO2, adhesion molecule with Ig like domain 2 .

$\begin{array}{r}\text { nuclear speck } \\ \text { nuclear matrix } \\ \text { chromosome }\end{array}$
$\begin{array}{r}\text { centrosome } \\ \text { nucleolus } \\ \text { cytosol }\end{array}$
cytoplasm
nucleus
nucleoplasm

Figure 6. GO enrichment analysis of differentially expressed genes in MCF-7 cells treated with MIH 2.4Bl compared with the control cells. The y-axis represents the top ten significantly enriched GO terms in each category defined by biological process, molecular function, and cellular component. The $\mathrm{x}$-axis data is presented in terms of the negative $\log$ (base 10) of the Benjamini-Hochberg adjusted P-values of each pathway. GO, gene ontology. 
Table III. KEGG pathways with significant enrichment of differentially expressed genes in MCF-7 cells treated with MIH 2.4B1.

\begin{tabular}{|c|c|c|c|c|c|}
\hline Pathway name & KEGG & P-value & Hit & Total genes & Percent \\
\hline FoxO signaling pathway & hsa04068 & 0.0037 & 35 & 134 & 26.1 \\
\hline Signaling pathways regulating pluripotency of stem cells & hsa04550 & 0.0121 & 34 & 140 & 24.3 \\
\hline Adherens junction & hsa04520 & 0.0161 & 21 & 71 & 29.6 \\
\hline RNA degradation & hsa03018 & 0.0138 & 22 & 77 & 28.6 \\
\hline TGF- $\beta$ signaling pathway & hsa04350 & 0.0149 & 23 & 84 & 27.4 \\
\hline Ubiquitin mediated proteolysis & hsa04120 & 0.0143 & 32 & 137 & 23.4 \\
\hline Protein processing in endoplasmic reticulum & hsa04141 & 0.0147 & 37 & 169 & 21.9 \\
\hline Insulin resistance & hsa04931 & 0.0279 & 26 & 108 & 24.1 \\
\hline Lysine degradation & hsa00310 & 0.0257 & 16 & 52 & 30.8 \\
\hline Transcriptional misregulation in cancer & hsa05202 & 0.0341 & 35 & 167 & 21.0 \\
\hline Endocytosis & hsa04144 & 0.0376 & 46 & 241 & 19.1 \\
\hline Renal cell carcinoma & hsa05211 & 0.0361 & 18 & 66 & 27.3 \\
\hline
\end{tabular}

Benjamini-Hochberg adjusted $\mathrm{P}<0.05$ was used for statistical significance. FoxO, Forkhead Box O; KEGG, Kyoto Encyclopedia of Genes and Genomes; TGF, transforming growth factor.

in regulating a wide variety of cellular processes, including cell cycle, apoptosis, regulation of autophagy, and oxidative stress resistance.

\section{Discussion}

Despite the existence of a considerable number of drugs for the treatment of cancer, in several cases, therapeutic success is not achieved due to treatment failures, resulting in high relapse rates, poor patient survival, or adverse effects. These outcomes necessitate a continuous search for novel more efficacious treatments with reduced side effects (37). Investigators have focused on studying the potential uses of mesoionic compounds due to of their unique structure and reaction properties, which make their biological activities particularly amenable to pharmaceutical use $(38,39)$. The synthesis of a new mesoionic compound, MIH 2.4Bl has been described in our previous study (4), and the selective cytotoxicity against the MCF-7 breast cancer cells was demonstrated in the present study.

A critical step in pharmaceutical development is to identify the direct targets of potential drug candidates (40). Mitochondria function as energy producers of energy in the form of ATP and as a result, have received attention as a potential target for novel cancer therapies (20). During physiological cell function, mitochondria produce ATP via oxidative phosphorylation, which constitutes the primary mechanism underlying the accumulation of (ROS. Due to the rapid proliferation rate of cancer cells, mitochondrial function in ROS production and oxidative stress serves a definitive role in carcinogenesis (41). Importantly, the dramatic reduction of mitochondrial respiration we observed in MCF-7 cells treated with $\mathrm{MIH} 2.4 \mathrm{Bl}$ in the present study may be associated with mitochondrial dysfunction resulting in ROS accumulation (42).

Autophagy is a fundamental mechanism of cellular homeostasis, initiated to protect cells by promoting turnover of organelles and proteins (43). Autophagy is also associated with the induction of programmed cell death (44), and also contributes to functions as a tumor suppressor by degrading damaged mitochondria that would otherwise contribute to ROS accumulation (45). In the present study, it was demonstrated that the mesoionic compound MIH2.4 Bl induced the expression of two autophagy-related proteins (Beclin-1 and ATG5), which serve an important role in the autophagic process (46) in response to stress caused by mitochondrial dysfunction. During the formation of autophagosomes, ATG5 is required for the conversion of LC3B-I to LC3B-II, a participant in autophagosome membrane expansion and fusion (47). Beclin 1 has been identified as a mammalian protein associated with autophagy and involved in cancer processes such as tumor suppression and cell death (48). Cheng et al (49) identified the role of autophagy in MCF-7 cells treated with Icariin (a flavonoid isolated from plants) by measuring the expression levels of ATG5, Beclin 1 and LC3-I to LC3-II proteins. Liang et al (50) demonstrated that exogenous Beclin 1 expression in MCF-7 cells resulted in decreased cell division and inhibition of tumorigenesis in a mouse model. Given the results of the present study, $\mathrm{MIH} 2.4 \mathrm{Bl}$ may have also induced autophagy in MCF-7 cells.

The results of mitochondrial respiration suggest a possible induction of cellular cytotoxicity by mitochondrial dysfunction induced by MIH 2.4Bl treatment. In support of these results, studies using the mesoionic compound SYD-1 in isolated rat liver mitochondria showed a functional decrease in electron transport and oxidative phosphorylation efficiency (19). Another mesoionic compound, MI-D, was also shown to act as an uncoupler of the respiratory chain between complexes II and III (9). Mechanistic studies demonstrated that alteration of membrane fluidity and elasticity were associated with the disruption of mitochondrial function by mesoionic compounds (10). Interestingly, altered mitochondrial metabolism has been suggested as an approach to overcome the resistance to apoptotic cell death observed in cancer cells (51).

Four genes that were shown to be highly upregulated and two genes that were highly downregulated based on the 
microarray data were selected for verification by RT-qPCR. Of the genes selected, ACRC (also known as germ cell nuclear acidic peptidase), is localized in the nucleus and serves a role in chromatin structure (52). ATF3 is a member of the mammalian ATF/CREB family of transcription factors that modulate TGF $\beta$ signaling in breast cancer (53). HBEGF is synthesized as a membrane-anchored glycoprotein that serves a role as a growth factor in breast cancer metastasis (54). RGS2 is a member of a family of genes that act as GTPase activating proteins for $\mathrm{G}$ proteins and may have an inhibitory effect in breast cancer (55). AMIGO2 is a member of a family of cell surface transmembrane proteins that function in cell adhesion and serves a survival role in cancer growth (56). DKK1 is a member of the Dickkopf family of proteins that are characterized by two cysteine-rich domains functioning to interact with the LRP6 co-receptor resulting in disruption of Wnt signaling (57). DKK1 has been suggested to have differential effects on lung and bone metastasis in breast cancer (58).

In conclusion, it was demonstrated that the mesoionic compound MIH 2.4Bl exhibits cytotoxic activity against MCF-7 breast cancer cells and reduced cell growth. Analysis of the functional effects of MIH 2.4B1 support a mechanism of action mediated by the induction of mitochondrial dysfunction and autophagy. Additional studies are required for elucidation of the specific mechanistic pathways involved in regulating autophagy. In particular, further studies are necessary to understand the specific role of different genes and pathways regulated by $\mathrm{MIH}$ 2,4B1. Nonetheless, these studies confirm the potential therapeutic use of MIH 2.4Bl for treatment of breast cancer.

\section{Acknowledgments}

The authors would like to thank Ms. Paula Polk in the Research Core Facility at LSU Health Shreveport for her technical assistance.

\section{Funding}

The present study was supported by Coordenação de Aperfeiçoamento de Pessoal de Nível Superior, through an international scholarship awarded through the Science without Borders program (grant no. 88887.122971/2016-00), and through research bridge funding provided by the Department of Cellular Biology and Anatomy at LSU School of Veterinary Medicine.

\section{Availability of data and materials}

The datasets used and/or analyzed during the present study are available from the corresponding author on reasonable request.

\section{Authors' contributions}

LAMC performed the experiments and analyzed and interpreted the data. DD assisted in designing and performing the cytotoxicity and OCR experiments and in interpreting the results. $\mathrm{ACH}$ assisted in designing and performing the autophagy experiments and in interpreting the results. SSA assisted in the statistical analysis of the data and in interpretation of the results. HDSS synthesized the mesoionic compound used in the experiments. PFAF directed the synthesis of the mesoionic compound used in the experiments and interpreted the analysis of the results. AW and MAGF assisted in designing the study and oversaw the analysis and interpretation of the data and wrote the manuscript. JMM directed the project, designed the study, oversaw the analysis and interpretation of the data, and assisted in writing the manuscript. All authors have read and approved the final manuscript.

\section{Ethics approval and consent to participate}

Not applicable.

\section{Patient consent for publication}

Not applicable.

\section{Competing interests}

The authors declare that they have no competing interests.

\section{References}

1. World Health Organization: WHO report on cancer: Setting priorities, investing wisely and providing care for all World Health Organization, 2020. https://apps.who.int/iris/ handle/10665/330745.

2. Harbeck N, Penault-Llorca F, Cortes J, Gnant M, Houssami N, Poortmans P, Ruddy K, Tsang J and Cardoso F: Breast cancer. Nat Rev Dis Primers 5: 66, 2019.

3. Santini D, Vincenzi B, Galluzzo S, Battistoni F, Rocci L, Venditti O, Schiavon G, Angeletti S, Uzzalli F, Caraglia M and Dicuonzo G: Repeated intermittent low-dose therapy with zoledronic acid induces an early, sustained, and long-lasting decrease of peripheral vascular endothelial growth factor levels in cancer patients. Clin Cancer Res 13: 4482-4486, 2007.

4. Bhosale SK, Deshpande SR, Wagh RD and Dhake AS: Biological activities of 1, 2, 3-oxadiazolium-5-olate derivatives. Der Chem Sin 6: 79-95, 2015.

5. Badami BV: Mesoionic compounds. Resonance 11: 40-48, 2006.

6 . Kier LB and Roche EB: Medicinal chemistry of the mesoionic compounds. J Pharm Sci 56: 149-168, 1967.

7. Senff-Ribeiro A, Echevarria A, Silva EF, Sanches Veiga S and Oliveira MB: Effect of a new 1,3,4-thiadiazolium mesoionic compound (MI-D) on B16-F10 murine melanoma. Melanoma Res 13: 465-471, 2003.

8. Senff-Ribeiro A, Echevarria A, Silva EF, Franco CR, Veiga SS and Oliveira MB: Cytotoxic effect of a new 1,3,4-thiadiazolium mesoionic compound (MI-D) on cell lines of human melanoma. Br J Cancer 91: 297-304, 2004.

9. Cadena SMSC, Carnieri EGS, Echevarria A and de Oliveira MBM: Effect of MI-D, a new mesoionic compound, on energy-linked functions of rat liver mitochondria. FEBS Lett 440: 46-50, 1998.

10. Cadena SMSC, Carnieri EGS, Echevarria A and de Oliveira MBM: Interference of MI-D, a new mesoionic compound, on artificial and native membranes. Cell Biochem Funct 20: 31-37, 2002.

11. Dunkley CS and Thoman CJ: Synthesis and biological evaluation of a novel phenyl substituted sydnone series as potential antitumor agents. Bioorg Med Chem Lett 13: 2899-2901, 2003.

12. Gozzi GJ, Pires Ado R, Martinez GR, Rocha ME, Noleto GR, Echevarria A, Canuto AV and Cadena SM: The antioxidant effect of the mesoionic compound SYD-1 in mitochondria. Chem Biol Interact 205: 181-187, 2013.

13. Galuppo LF, dos Reis Lívero FA, Martins GG, Cardoso CC, Beltrame OC, Klassen LMB, Canuto AV, Echevarria A, Telles JE, Klassen G and Acco A: Sydnone 1: A mesoionic compound with antitumoral and haematological effects in vivo. Basic Clin Pharmacol Toxicol 119: 41-50, 2016. 
14. Amaral de Mascena Costa L, Cássio Silva de Lima F, da Silva Viana R, de Sousa Araújo S, Wischral A, Diógenes da Silva Souzad H, Filgueiras de Athayde Filhod P, Araújo de Azevedo L, Alves Junior S, Adrião Gomes Filho M and Mathis JM: Abstract 5877: Antitumor activity of the mesoionic compound MI H 2.4 on breast cancer cell lines. Cancer Res 78 (Suppl 13), 2018.

15. Lee AV, Oesterreich S and Davidson NE: MCF-7 cells-changing the course of breast cancer research and care for 45 years. J Natl Cancer Inst 107: djv073, 2015

16. Comşa Ş, Cîmpean AM and Raica M: The story of MCF-7 breast cancer cell line: 40 years of experience in research. Anticancer Res 35: 3147-3154, 2015.

17. Anvar SY, Allard G, Tseng E, Sheynkman GM, de Klerk E, Vermaat M, Yin RH, Johansson HE, Ariyurek Y, den Dunnen JT and Turner SW: Full-length mRNA sequencing uncovers a widespread coupling between transcription initiation and mRNA processing. Genome Biol 19: 46, 2018

18. Chiang YS, Huang YF, Midha MK, Chen TH, Shiau HC and Chiu KP: Single cell transcriptome analysis of MCF-7 reveals consistently and inconsistently expressed gene groups each associated with distinct cellular localization and functions. PLoS One 13: e0199471, 2018.

19. Halila GC, de Oliveira MB, Echevarria A, Belém AC, Rocha ME, Carnieri EG, Martinez GR, Noleto GR and Cadena SM: Effect of sydnone SYD-1, a mesoionic compound, on energy-linked functions of rat liver mitochondria. Chem Biol Interact 169: 160-170, 2007.

20. Weinberg SE and Chandel NS: Targeting mitochondria metabolism for cancer therapy. Nat Chem Biol 11: 9-15, 2015.

21. Kubli DA and Gustafsson ÅB: Mitochondria and mitophagy: The yin and yang of cell death control. Circ Res 111: 1208-1221, 2012.

22. Lira BF, de Athayde Filho PF, Miller J, Simas AM, de Farias Dias A and Vieira MJ: Synthesis and characterization of some new mesoionic 1,3-thiazolium-5-thiolates via cyclodehydration and in situ 1, 3-dipolar Cycloaddition/cycloreversion Molecules 7: 791-800, 2002.

23. Feoktistova M, Geserick P and Leverkus M: Crystal violet assay for determining viability of cultured cells. Cold Spring Harbor Protoc 2016: pdb-rot087379, 2016.

24. Tsou SH, Chen TM, Hsiao HT and Chen YH: A critical dose of doxorubicin is required to alter the gene expression profiles in MCF-7 cells acquiring multidrug resistance. PLoS One 10: e0116747, 2015.

25. Jokar F, Mahabadi JA, Salimian M, Taherian A, Hayat SMG, Sahebkar A and Atlasi MA: Differential expression of HSP90 in MDA-MB-231 and MCF-7 cell lines after treatment with doxorubicin. J Pharmacopuncture 22: 28-34, 2019

26. Fornari FA, Randolph JK, Yalowich JC, Ritke MK and Gewirtz DA: Interference by doxorubicin with DNA unwinding in MCF-7 breast tumor cells. Mol Pharmacol 45: 649-656, 1994.

27. Huang DW, Sherman BT and Lempicki RA: Systematic and integrative analysis of large gene lists using DAVID bioinformatics resources. Nat Protoc 4: 44-57, 2009.

28. Huang DW, Sherman BT and Lempicki RA: Bioinformatics enrichment tools: Paths toward the comprehensive functional analysis of large gene lists. Nucleic Acids Res 37: 1-13, 2009.

29. Ashburner M, Ball CA, Blake JA, Botstein D, Butler H, Cherry JM, Davis AP, Dolinski K, Dwight SS, Eppig JT and Harris MA: Gene ontology: Tool for the unification of biology. Nat Genet 25: 25-29, 2000.

30. The Gene Ontology Consortium: The gene ontology resource: 20 years and still GOing strong. Nucleic Acids Res 47: D330-D338, 2019

31. Kanehisa M: Post-genome informatics. Oxford University Press, Oxford, 2000.

32. Benjamini Y and Hochberg Y: Controlling the false discovery rate: A practical and powerful approach to multiple testing. J R Stat Soc Series B (Methodological) 57: 289-300, 1995.

33. Livak KJ and Schmittgen TD: Analysis of relative gene expression data using real-time quantitative PCR and the 2(-Delta Delta C(T)) method. Methods 25: 402-408, 2001

34. Kabeya Y, Mizushima N, Ueno T, Yamamoto A, Kirisako T, Noda T, Kominami E, Ohsumi Y and Yoshimori T: LC3, a mammalian homologue of yeast Apg8p, is localized in autophagosome membranes after processing. EMBO J 19: 5720-5728, 2000.

35. Sica V, Bravo-San Pedro JM, Stoll G and Kroemer G: Oxidative phosphorylation as a potential therapeutic target for cancer therapy. Int J Cancer 146: 10-17, 2020.

36. Brand MD and Nicholls DG: Assessing mitochondrial dysfunction in cells. Biochem J 435: 297-312, 2011.
37. Tang Y, Wang Y, Kiani MF and Wang B: Classification, treatment strategy, and associated drug resistance in breast cancer. Clin Br Cancer 16: 335-343, 2016.

38. Kaur G and Singh R: Thiadiazole analogs as potential pharmacological agents: A brief review. Int J Pharm Sci 6: 35-46, 2014.

39. Abdualkader AM, Taher MU and Yusoff NI: Mesoionic sydnones. A review in their chemical and biological properties. Int J Pharm Pharm Sci 9: 1-9, 2017.

40. Rogers GW, Burroughs SE and Dranka BP: Direct measurements of cellular metabolism for identification of mitochondrial drug targets. Agilent Technologies: 14 Nov, 2018.

41. Czupiela PP, Delplace V and Shoicheta MS: Cationic block amphiphiles show anti-mitochondrial activity in multi-drug resistant breast cancer cells. J Control Realeas 305: 210-219, 2019.

42. Storz P: Reactive oxygen species in tumor progression. Front Biosci 10: 1881-1896, 2005

43. Mizushima N, Levine B, Cuervo AM and Klionsky DJ: Autophagy fights disease through cellular self-digestion. Nature 451: 1069-1075, 2008

44. Pampliega O, Orhon I, Patel B, Sridhar S, Diaz-Carretero A, Beau I, Codogno P, Satir BH, Satir P and Cuervo AM: Functional interaction between autophagy and ciliogenesis. Nature 502: 194-200, 2013

45. Matsuda N, Sato S, Shiba K, Okatsu K, Saisho K, Gautier CA, Sou YS, Saiki S, Kawajiri S, Sato F, et al: PINK1 stabilized by mitochondrial depolarization recruits Parkin to damaged mitochondria and activates latent Parkin for mitophagy. J Cell Biol 189: 211-221, 2010.

46. Akar U, Chaves-Reyez A, Barria M, Tari A, Sanguino A, Kondo Y, Kondo S, Arun B, Lopez-Berestein G and Ozpolat B: Silencing of Bcl-2 expression by small interfering RNA induces autophagic cell death in MCF-7 breast cancer cells. Autophagy 4 : 669-679, 2008

47. Nakagawa I, Amano A, Mizushima N, Yamamoto A, Yamaguchi $\mathrm{H}$ and Kamimoto T: Autophagy defends cells against invading group A Streptococcus. Science 306: 1037-1040, 2004.

48. He C and Levine B: The Beclin 1 interactome. Curr Opin Cell Biol 22: 140-149, 2010.

49. Cheng X, Tan S, Duan F, Yuan Q, Li Q and Deng G: Icariin induces apoptosis by suppressing autophagy in Tamoxifen-resistant breast cancer cell line MCF-7/TAM Breast Cancer 26: 766-775, 2019.

50. Liang XH, Jackson S, Seaman M, Brown K, Kempkes B Hibshoosh H and Levine B: Induction of autophagy and inhibition of tumorigenesis by beclin 1. Nature 402: 672-676, 1999.

51. Vyas S, Zaganjor E and Haigis MC: Mitochondria and cancer. Cell 166: 555-566, 2016.

52. Bhargava V, Goldstein CD, Russell L, Xu L, Ahmed M, Li W, Casey A, Servage K, Kollipara R, Picciarelli Z and Kittler R: GCNA preserves genome integrity and fertility across species. Dev Cell 52: 38-52, 2020

53. Yin X, Wolford CC, Chang YS, McConoughey SJ, Ramsey SA, Aderem A and Hai T: ATF3, an adaptive-response gene, enhances TGF $\beta$ signaling and cancer-initiating cell features in breast cancer cells. J Cell Sci 123: 3558-3565, 2010.

54. Zhou ZN, Sharma VP, Beaty BT, Roh-Johnson M, Peterson EA, Van Rooijen N, Kenny PA, Wiley HS, Condeelis JS and Segall JE: Autocrine HBEGF expression promotes breast cancer intravasation, metastasis and macrophage-independent invasion in vivo. Oncogene 33: 3784-3793, 2014.

55. Lyu JH, Park DW, Huang B, Kang SH, Lee SJ, Lee C, Bae YS, Lee JG and Baek SH: RGS2 suppresses breast cancer cell growth via a MCPIP1-dependent pathway. J Cell Biochem 116: 260-267, 2015.

56. Fontanals-Cirera B, Hasson D, Vardabasso C, Di Micco R, Agrawal P, Chowdhury A, Gantz M, de Pablos-Aragoneses A, Morgenstern A, Wu P, et al: Harnessing BET inhibitor sensitivity reveals AMIGO2 as a melanoma survival gene. Mol Cell 68 731-744.e9, 2017.

57. Niida A, Hiroko T, Kasai M, Furukawa Y, Nakamura Y, Suzuki Y, Sugano S and Akiyama T: DKK1, a negative regulator of Wnt signaling, is a target of the beta-catenin/TCF pathway. Oncogene 23: 8520-8526, 2004.

58. Zhuang X, Zhang H, Li X, Li X, Cong M, Peng F, Yu J, Zhang X, Yang Q and Hu G: Differential effects on lung and bone metastasis of breast cancer by Wnt signalling inhibitor DKK1. Nat Cell Biol 19: 1274-1285, 2017.

This work is licensed under a Creative Commons Attribution-NonCommercial-NoDerivatives 4.0 International (CC BY-NC-ND 4.0) License. 